SECTION 2. Applied mathematics. Mathematical modeling.

Yertayev Karabek Yertayevich

doctor of economic Sciences,

Head of the chair "Economy and evaluation"

Taraz State University named after M.Kh. Dulati,

Kazakhstan

Shevtsov Alexandr Nikolayevich candidate of technical Sciences,

President of International Academy of Theoretical \& Applied Sciences,

Kazakhstan

\title{
ON SOME ALGORITHMS FOR CONSTRUCTING NON-STANDARD CHARTS
}

Abstract: In this article are developed algorithms of complex graphs to a method of analysis of economic data in quadrants.

Key words: algorithms, program, economic parameters.

\section{О НЕКОТОРЫХ АЛГОРИТМАХ ПОСТРОЕНИЯ НЕСТАНДАРТНЫХ ГРАФИКОВ}

\begin{abstract}
Аннотация: В данной статье разрабатьваются алгоритмы построения сложных графиков для метода анализа экономических данных по квадрантам.

Ключевые слова: алгоритмы, программа, экономические параметры.
\end{abstract}

Во многих экономических задачах и анализе данных возникает необходимость анализа одновременно по нескольким параметрам. При исследовании данных - чаще всего параметры имеют положительные значения, поэтому будем откладывать только положительные значения как в положительных направлениях осей, так и в отрицательных.

Рассмотрим задачу с четырьмя параметрами, и распределением по годам:

Таблица 1

\section{Экономические параметры}

\begin{tabular}{|c|c|c|c|c|}
\hline Годы & $\begin{array}{c}\text { Индекс оплаты } \\
\text { труда }\end{array}$ & Индекс прибыли & $\begin{array}{c}\text { Индекс основного } \\
\text { капитала }\end{array}$ & $\begin{array}{c}\text { Индекс численности } \\
\text { занятых }\end{array}$ \\
\hline $\mathbf{2 0 0 0}$ & 1 & 1 & 1 & 1 \\
\hline $\mathbf{2 0 0 1}$ & 1,252567 & 1,295401 & 1,1770239 & 1,080277375 \\
\hline $\mathbf{2 0 0 2}$ & 1,327839 & 1,473967 & 1,4352696 & 1,081906144 \\
\hline $\mathbf{2 0 0 3}$ & 1,629062 & 1,845689 & 1,6777588 & 1,126463474 \\
\hline $\mathbf{2 0 0 4}$ & 2,098224 & 2,382348 & 1,9484685 & 1,158168037 \\
\hline $\mathbf{2 0 0 5}$ & 2,691298 & 3,051133 & 2,4769534 & 1,170940171 \\
\hline $\mathbf{2 0 0 6}$ & 3,465393 & 4,422141 & 3,3740079 & 1,193920335 \\
\hline $\mathbf{2 0 0 7}$ & 4,758329 & 5,143869 & 4,0987124 & 1,230607966 \\
\hline $\mathbf{2 0 0 8}$ & 5,643622 & 6,812456 & 5,1825798 & 1,267085954 \\
\hline
\end{tabular}




\begin{tabular}{|c|c|c|c|c|}
$\mathbf{2 0 0 9}$ & 6,187547 & 7,292092 & 6,5727556 & 1,274536365 \\
\hline $\mathbf{2 0 1 0}$ & 8,041124 & 9,208001 & 6,9501734 & 1,308530882 \\
\hline $\mathbf{2 0 1 1}$ & 9,901195 & 11,79825 & 8,6424834 & 1,338751814 \\
\hline $\mathbf{2 0 1 2}$ & & & & 1,37189163 \\
\hline
\end{tabular}

Распределим параметры, соответственно, по осям Индекс прибыли Ох, Индекс оплаты труда $\sim$ Оу, Индекс численности занятых $~$ противоположно Ох, Индекс основного капитала противоположно Оу. Разработаем алгоритмы на Delphi XE4, для построения данного графика, причем все данные будем загружать в программу из Exel (рис.1).

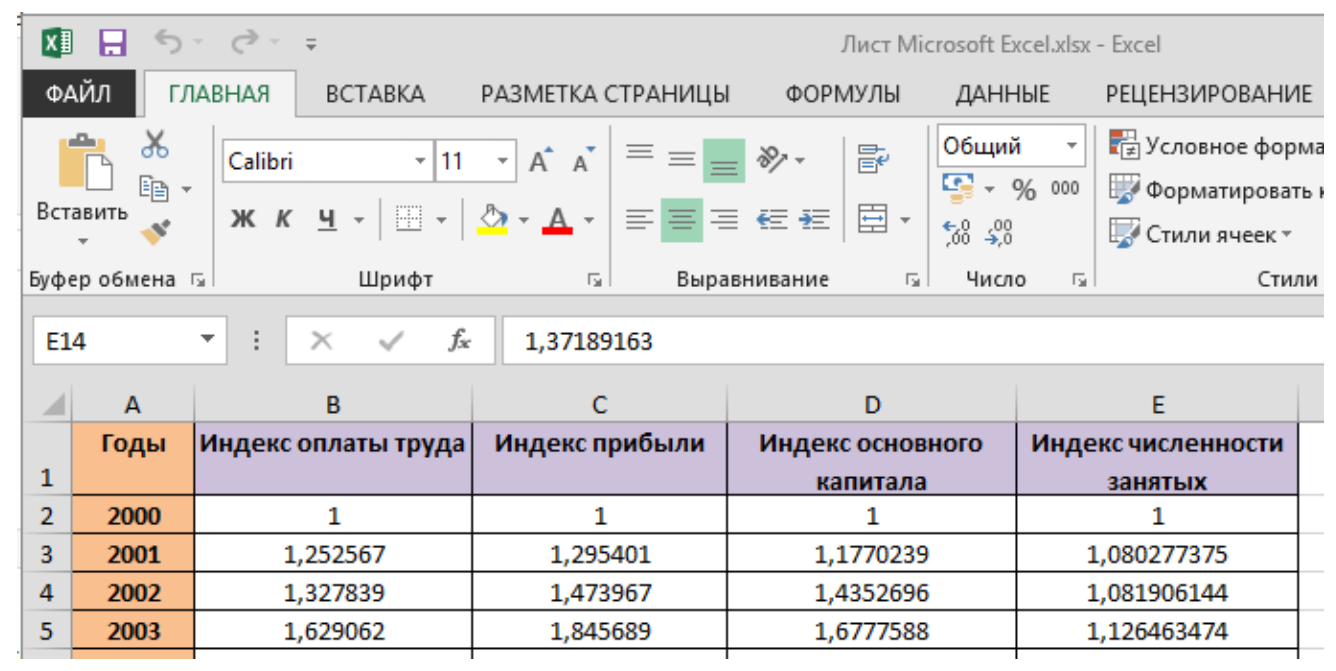

Рисунок 1 - Исходные данные.

Таблица 2

Код программы

const $\mathrm{ABCD}=$ 'BCDE';

var Form1: TForm1;

se, $\mathrm{x} 0, \mathrm{y} 0, \mathrm{~s} 0$,m:double;

a,b,c,d:array[1..2] of array[1..100]of double;

z:array[1..4,1..100] of double;

i,j,n,ii,jj:integer;

s:array[1..4] of array[0..100] of string;

bb:boolean;

mi:array[1..5,1..3] of integer;

implementation

$\{\$ \mathrm{R} * . \mathrm{dfm}\}$

procedure TForm1.BitBtn1Click(Sender: TObject);

begin

try // Create a Word Instance

ExcelApp := CreateOleObject('Excel.Application');

except // Error...

Exit;

end;

ExcelApp.Workbooks.Open(label1.Caption,0,readOnly:=false);//<имя файла>,0,True);

ExcelApp.Visible := false; 
ExcelApp.DisplayAlerts := False;

$\mathrm{n}:=-1$;

bb:=true;

while bb do

begin

inc(n);

se: $=0$;

for $\mathrm{I}:=1$ to 4 do

$\mathrm{s}[\mathrm{i}][\mathrm{n}]:=$ ExcelApp.Range[ABCD[i]+inttostr(n+1)];// ExcelApp.Range['B17'];

if $n>1$ then

if (s[1][n]=")or(s[2][n]=")or(s[3][n]=")or(s[4][n]=") then

bb:=false;

end;

ExcelApp.Workbooks.Close; ExcelApp.Quit;

$\mathrm{n}:=\mathrm{n}-1 ; \quad \mathrm{m}:=0$;

for $\mathrm{I}:=1$ to $\mathrm{n}$ do

begin

if strtofloat(s[1][i])>m then $\mathrm{m}$ :=strtofloat(s[1][i]);

if strtofloat(s[2][i]) $>\mathrm{m}$ then $\mathrm{m}:=\operatorname{strtofloat}(\mathrm{s}[2][\mathrm{i}])$;

if strtofloat(s[3][i]) $>\mathrm{m}$ then $\mathrm{m}$ :=strtofloat(s[3][i]);

if strtofloat(s[4][i])>m then $\mathrm{m}$ :=strtofloat(s[4][i]);

a[1][i]:=strtofloat(s[2][i]);

$\mathrm{a}[2][\mathrm{i}]:=$ strtofloat(s[1][i]);

series1.AddXY(a[1][i],a[2][i]);

$\mathrm{b}[1][\mathrm{i}]:=-$ strtofloat(s[4][i]);

series2.AddXY(b[1][i],b[2][i]);

$\mathrm{b}[2][\mathrm{i}]:=$ strtofloat(s[1][i]);

c[1][i]:=-strtofloat(s[4][i]);

c[2][i]:=-strtofloat(s[3][i]);

series3.AddXY(c[1][i],c[2][i]);

d[2][i]:=-strtofloat(s[3][i]);

$\mathrm{d}[1][\mathrm{i}]:=$ strtofloat(s[2][i]);

series4.AddXY(d[1][i],d[2][i]);

end;

$\mathrm{x} 0:=\operatorname{int}(\mathrm{m})+1 ; \mathrm{y} 0:=\operatorname{int}(\mathrm{m})+1$;

series5.AddXY(-x0,-y0); series5.AddXY $(\mathrm{x} 0, \mathrm{y} 0)$;

series6.AddXY(-x0,y0); series6.AddXY $(\mathrm{x} 0,-\mathrm{y} 0)$;

series7.AddXY(-x0,0); series7.AddXY $(\mathrm{x} 0,0)$;

series8.AddXY(0,-y0); series8.AddXY(0,y0);

chart1.SaveToBitmapFile('График.bmp');

image1.Picture.LoadFromFile('График.bmp');

image2.Canvas.CopyRect(Rect(90,30,image1.Picture.Width+90,image1.Picture.Height+ 30),

image1.Canvas,

Rect(0,0,image1.Picture.Width,image1.Picture.Height));

image2.Canvas.Font.Style:=[fsbold];

image2.Canvas.TextOut(600,300,s[2][0]); 
image2.Canvas.TextOut(60,300,s[4][0]);

image2.Canvas.TextOut(320,20,s[1][0]);

image2.Canvas.TextOut(320,530,s[3][0]);

for $\mathrm{i}:=100$ to trunc(image2.Width/2) do

for $\mathrm{j}:=$ trunc(image2.Height/2) to image2.Height do

begin

for ii := 1 to 5 do for $\mathrm{jj}:=1$ to 3 do

begin

if image2.Canvas.Pixels $[\mathrm{i}+\mathrm{ii}, \mathrm{j}+\mathrm{jj}]=$ clblack then mi[ii,jj]:=1;

if image2.Canvas.Pixels[i+ii,j+jj] $=$ clwhite then mi[ii,jj]:=0;

end;

if

$(\mathrm{mi}[1,1]+\mathrm{mi}[2,1]+\mathrm{mi}[3,1]+\mathrm{mi}[4,1]+\mathrm{mi}[5,1]+\mathrm{mi}[5,2]+\mathrm{mi}[5,3]+\mathrm{mi}[4,3]+\mathrm{mi}[3,3]+\mathrm{mi}[2,3]+$ $\mathrm{mi}[1,3]+\mathrm{mi}[1,2]=0)$ and $(\mathrm{mi}[2,2]+\mathrm{mi}[3,2]+\mathrm{mi}[4,2]=3)$ then

begin

image2.Canvas.Pixels[i+2,j+2]:=clwhite;

image2.Canvas.Pixels[i+3,j+2]:=clwhite;

image2.Canvas.Pixels[i+4,j+2]:=clwhite;

end; end;

image2.Picture.SaveToFile('График+++.bmp');

end;

procedure TForm1.FormCreate(Sender: TObject);

begin

DragAcceptFiles(Handle,True);

end;

procedure TForm1.WMDropFiles(var Msg: TMessage);

Var

Filename: array[0..256] of char;

begin

DragQueryFile(THandle(Msg.WParam),0,Filename,SizeOf(Filename));

label1.caption:=FileName;

label2.caption:=extractfilename(label1.caption);

BitBtn1.Click;

DragFinish(THandle(Msg.WParam)); end; end. 


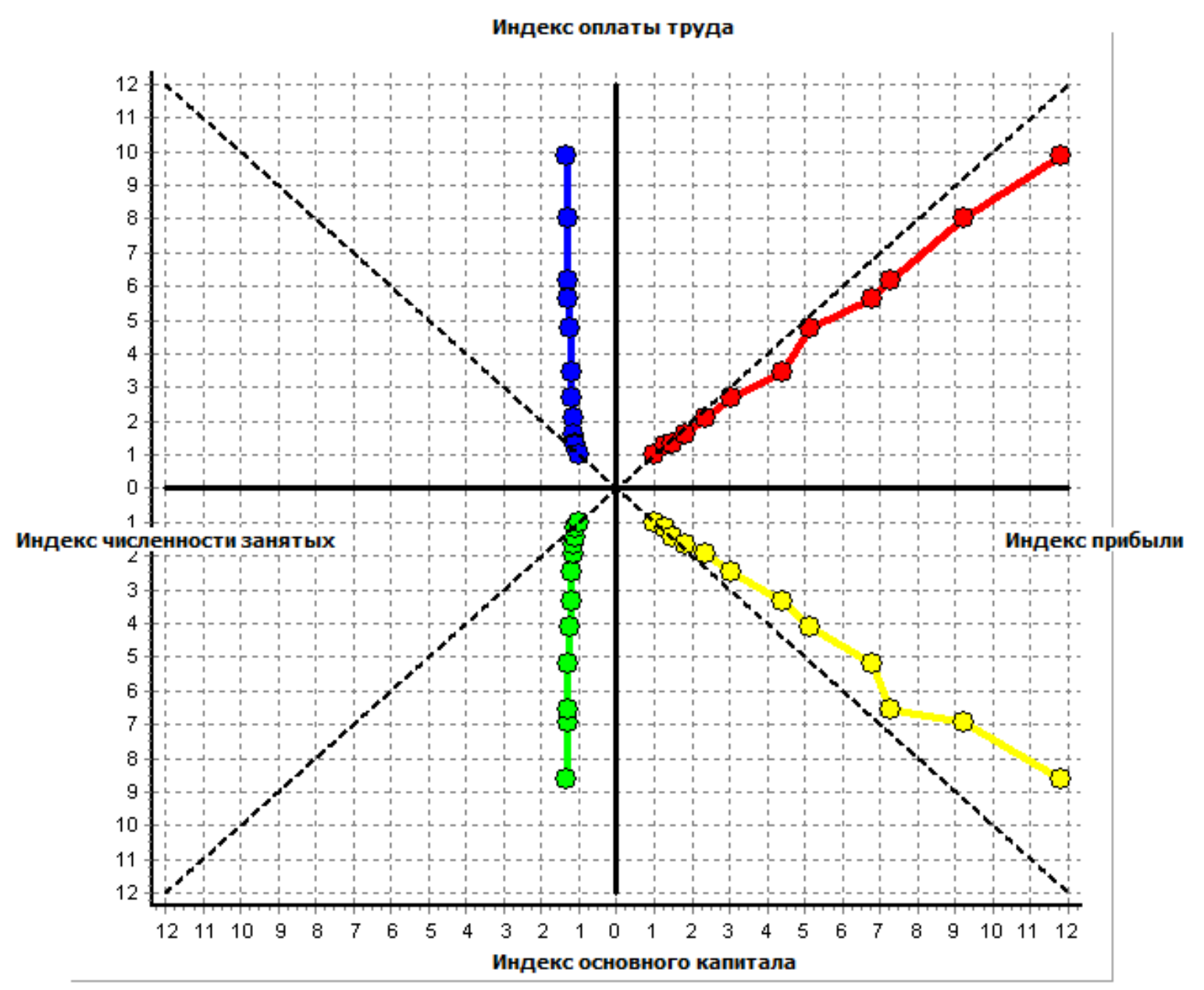

Рисунок 2 - Полученный график.

В результате реализации разработанных алгоритмов получим следующий график (рис.2). Особенностью метода анализа данных является оценка полученных кривых относительно биссектрис углов и выделения именно тех кривых, которые лежат по одну сторону от соответствующих биссектрис в своем квадранте. В настоящее время метод используется для прогнозов дальнейшего изменения и экстраполяции индексов и других параметров в совокупности.

\section{Литература}

1. Коренкова С.И. Экономический анализ (Анализ хозяйственной деятельности): Учебное пособие для студентов специальностей “Финансы и кредит”, “Налоги и налогообложение”, “Менеджмент организаций”. Тюмень: Издательство Тюменского государственного университета, 2008 г., 331 с.

2. Экономический анализ. [Электронный ресурс]. URL http://vsempomogu.ru/economika/ahd/12-5.html (дата обращения: 20.12.2013).

3. Основные приемы экономического анализа. [Электронный ресурс]. URL http://otherreferats.allbest.ru/economy/00029057_0.html (дата обращения: 20.12.2013).

4. СеЛЕВИч Т.С. ОСОБЕННОСТИ ТЕКУЩЕГО И СТРАТЕГИЧЕСКОГО КОНКУРЕНТНОГО АНАЛИЗА. Томский политехнический университет. [Электронный ресурс]. URL: http://selevich.professorjournal.ru/c/document_library/get_file?uuid=18a4cc2e-2ff144e0-8fbb-0b8997ec8540\&groupId=506886 (дата обращения: 20.12.2013). 